Canadian University Music Review

Canadian University Music Review

Revue de musique des universités canadiennes

\title{
Stratégies citationnelles dans Die Soldaten de Bernd Alois Zimmermann
}

\section{Lise Viens}

Volume 17, numéro 1, 1996

URI : https://id.erudit.org/iderudit/1014690ar

DOI : https://doi.org/10.7202/1014690ar

Aller au sommaire du numéro

\section{Éditeur(s)}

Canadian University Music Society / Société de musique des universités canadiennes

\section{ISSN}

0710-0353 (imprimé)

2291-2436 (numérique)

Découvrir la revue

Citer cet article

Viens, L. (1996). Stratégies citationnelles dans Die Soldaten de Bernd Alois Zimmermann. Canadian University Music Review / Revue de musique des universités canadiennes, 17(1), 1-19. https://doi.org/10.7202/1014690ar
Résumé de l'article

L'auteure examine trois extraits de l'opéra Die Soldaten (1958-60, 1962-64) de Bernd Alois Zimmermann et montre comment la citation est venue fournir des solutions aux problèmes compositionnels rencontrés la dans conception d'une œuvre dramaturgique à ce moment crucial dans l'évolution vers la

formulation du pluralisme musical, technique compositionnelle définie par le compositeur vers 1960 et visant à traduire une conception sphérique du temps. Ces trois passages sont la deuxième scène du deuxième acte (janvier 1960), où l'insertion d'un choral de Bach est venue résoudre le problème de la représentation simultanée de trois scènes, « Intermezzo » du même acte (avril 1963), privilégiant également un choral de Bach comme fondement du complexe (à la manière d'un cantus firmus) et enfin, le " Preludio " (novembre 1962), où la démultiplication du Dies Irae en canon proportionnel révèle à la fois la fascination de Zimmermann pour les techniques polyphoniques franco-flamandes et l'importance structurelle qu'accorde le compositeur aux passages citants, ici non plus au centre d'une structure tripartite comme dans les deux cas précédents, mais comme aboutissement du processus dramaturgique.
All Rights Reserved (C Canadian University Music Society / Société de musique des universités canadiennes, 1996
Ce document est protégé par la loi sur le droit d'auteur. L'utilisation des services d'Érudit (y compris la reproduction) est assujettie à sa politique d'utilisation que vous pouvez consulter en ligne.

https://apropos.erudit.org/fr/usagers/politique-dutilisation/ 


\title{
STRATÉGIES CITATIONNELLES DANS DIE SOLDATEN DE BERND ALOIS ZIMMERMANN
}

\author{
Lise Viens
}

Reliée par de solides ancrages au mouvement d'avant-garde, l'œuvre de Bernd Alois Zimmermann (1918-70) a pourtant conservé le caractère d'une entreprise solitaire'. Une prédilection pour l'emprunt dès le début de sa production au début des années 40 et l'usage "non-orthodoxe » de citations ${ }^{2}$ dans des œuvres sérielles du début des années 50 ont certainement contribué à accentuer cette réalité chez ce compositeur qui se décrivait comme "l'aîné de ce qu'il est convenu d'appeler la troisième génération de la nouvelle musique $»^{3}$. Cette tendance à intégrer la résonance de matériaux venus du dehors caractérise chacune des quatre périodes créatrices qui se dessinent dans l'ensemble de la production de Zimmermann. La visée des procédures citationnelles et, par conséquent, la fonction des objets empruntés dans l'œuvre connaîtront cependant des modifications importantes.

Dans la première période (les années 40), il s'agira surtout de l'intégration d'objets stylistiques, tels le jazz ou des danses populaires et ce, selon des procédés de stylisation issus d'une fascination avouée pour Stravinsky et Milhaud $^{4}$. Cette première période voit aussi les premières manifestations de la

\footnotetext{
1 Une première version de ce texte a été présentée au congrès 1995 de la Société de musique des universités canadiennes (Montréal) et a remporté le prix George-Proctor.

2 C'est ainsi que Zimmermann qualifie l'usage de la citation d'un motif de la Symphonie en do de Stravinsky dans son Concerto pour hautbois (1952). Il écrit à Reinhold Schubert : « je me suis permis ici un geste très désapprouvé des orthodoxes [...] » («Ich habe mir den von den Orthodoxen sehr mißbilligten Kunstgriff erlaubt, im ersten Satz des Oboen-Konzertes innerhalb des Zwölftonverfahrens Stravinsky-Zitate organisch einzubauen »); lettre de Zimmermann à Reinhold Schubert, 16 janvier 1957, citée par Wulf Konold, Bernd Alois Zimmermann : der Komponist und sein Werk (Cologne : DuMont Verlag, 1986), 31.

3Lettre de Zimmermann à Monika Lichtenfeld, 27 novembre 1966, dont des extraits sont publiés dans Bernd Alois Zimmermann (1918-1970) : Dokumente zu Leben und Werk, documents réunis et commentés par Klaus Ebbeke (Berlin : Akademie der Künste, 1989), 11. Le caractère paradoxal de la position de Zimmermann vis-à-vis de cette génération est particulièrement frappant dans le passage suivant, extrait d'un article publié en 1961 : «L'auteur appartient à la catégorie des jeunes compositeurs et il s'est laissé convaincre que cette désignation ne concerne aucunement l'âge [...] mais les convictions ; et dans le cas de l'auteur, les opinions stylistiques telles qu'elles sont définies par le cercle des jeunes compositeurs de l'école de Darmstadt. L'auteur vit comme compositeur indépendant. Ce qui, pour un jeune compositeur, semble tout d'abord surprenant, est enregistré par de nombreux critiques comme prétention monstrueuse, ou comme la preuve que les miracles existent. » Bernd Alois Zimmermann, « Musiciens d'aujourd'hui » [1961], traduit par Edna Politi, Contrechamps 5 (1985) : 30.

4 On pense par exemple à l'insertion du folklore brésilien, pensé comme « la représentation qu'ont les Européens de ce folklore " (Zimmermann) dans le ballet Alagoana (œuvre composée entre 1951 et 1955 mais dont l'origine remonte, selon le compositeur, au début des années 40) ou encore à l'ennoblissement d'un rythme de rumba dans une forme classique comme le rondo du Concerto pour violon (1950).
} 
citation en tant qu' hommage rendu aux maîtres dans des œuvres où l'on décèle à la fois un désir d'établir une filiation et le caractère d'une lutte éclairée et consciente avec les pères. Au cours de la deuxième période, qui correspond à la première moitié des années 50 , les citations seront principalement intégrées à la fin d'un processus de genèse téléologique désormais sériel ${ }^{5}$. Par exemple, la citation d'une mélodie de choral complète dans la dernière des 12 sections de la Sonate pour alto solo (1955), où chacune des 11 premières sections est imprégnée de réminiscences anticipées du choral. À partir de la troisième période créatrice, dite " pluraliste » selon les propres termes du compositeur, le statut de la citation subit une modification d'envergure. Il ne s'agit plus de faire le « portrait » d'un objet stylistique - avec toute la notion de distanciation que cela implique - ni d'intégrer une citation unique au discours ambiant. Sans délaisser le caractère fondamental des premières stratégies, Zimmermann les transmue en une présentation sans détour du réel dans l'œuvre. Les citations, désormais fragmentaires et littéralement insérées, font maintenant irruption à des endroits précis, soit dans le collage de citations multiples, soit dans un passage où une seule citation est démultipliée en canon de proportion rythmique. Le but de la citation est maintenant de favoriser la perception de strates temporelles hétérogènes, objectif qui constitue un des points principaux de la technique compositionnelle zimmermannienne dite pluraliste.

Plusieurs facteurs sont à l'origine de cette modification du statut de la citation. Je propose ici d'envisager cette question à travers l'examen de trois des principaux passages citants de l'opéra Die Soldaten : le centre de la deuxième scène de l'acte 2 , le centre de l' «Intermezzo » du même acte et le "Preludio ». La genèse et la facture de ces trois passages montrent comment une redéfinition des stratégies citationnelles est venue fournir des solutions aux problèmes rencontrés dans la conception d'une œuvre dramaturgique à ce moment crucial dans l'évolution du langage zimmermannien. La chronologie des Soldats montre qu'en ce sens, la représentation de trois scènes simultanées dans la deuxième scène de l'acte $2 \mathrm{a}$ été particulièrement déterminante dans le cheminement compositionnel de Zimmermann vers le pluralisme.

\section{Chronologie}

La genèse des Soldats est résumée au tableau $1^{6}$. Cette genèse montre deux phases compositionnelles principales : la première de 1958 à 1960 et la deuxième

\footnotetext{
5Dans la nomenclature des quatre catégories d'emprunt qui se dégagent de l'analyse du corpus zimmermannien (stylisation, hommage, genèse téléologique et pluralisme), j'ai emprunté l'expression genèse téléologique à James Hepokoski, qui décrit un procédé citationnel chez Charles Ives dont l'intention et la méthode sont proches des visées de Zimmermann. Voir James Hepokoski, « Temps perdu, " The Musical Times 135, $\mathrm{n}^{\circ} 1822$ (1994) : 746-51, et le chapitre 1 (« Catégories citationnelles dans l'évolution de la pensée créatrice zimmermannienne ») de ma thèse de doctorat " La citation dans la pensée créatrice de Bernd Alois Zimmermann » (McGill University, 1995), 24-35.

6 Ce résumé de la genèse des Soldats a été établi à l'aide des données fournies par Wilfried Gruhn, «Zur Entstehungsgeschichte von Bernd Alois Zimmermanns Oper Die Soldaten », Die Musikforschung 38, n” 1 (1985) : 8-15, Klaus Ebbeke, "La genèse des Soldats », traduit par Carlo Russi, Musica 88 : Die Soldaten / Bernd Alois Zimmermann, édité par Laurence Helleu (Strasbourg : Musica 88, Dernières nouvelles d'Alsace, Contrechamps, 1988), 15-25, et Wulf Konold, Bernd Alois Zimmermann : der
} 
de 1963 à 1965. Ces deux phases engendrent, à l'intérieur même de l'œuvre achevée, une coexistence stylistique tout à fait saisissable pour l'auditeur attentif. Lors de la première étape, Zimmermann ébauche d'abord un plan général de l'opéra en 13 scènes distribuées en trois actes. Aux 12 premières scènes correspondent 12 séries différentes, déduites - par voie de permutation - d'un matériau unique : une série contenant tous les intervalles. Pendant cette première étape, Zimmermann achèvera, dans l'ordre, les cinq scènes de l'acte 1 (terminées en avril 1959), les trois premières scènes de l'acte 3 (terminées en octobre 1959) et, enfin, les deux scènes de l'acte 2 (scène 1 : novembre 1959 ; scène 2 : janvier 1960). C'est après la composition de la deuxième scène de l'acte 2, qualifiée, encore en 1964, de « point culminant en difficulté scénique et musicale ${ }^{7}$, que le compositeur interrompt son travail sur les Soldats dont la création avait été projetée pour juin 1960.

On peut s'interroger sur les motifs de cette interruption qui durera trois ans. On sait que l'Opéra de Cologne refuse brusquement de produire l'ouvrage à la date prévue en raison, entre autres, de sa prétendue inexécutabilité, et ce bien que les Soldats ne soient pas encore la gigantesque production que l'Opéra de Cologne créera enfin le 15 février 1965. Cette production, on le sait, inclut 17 chanteurs, 8 acteurs, des danseurs, quelques 100 musiciens, 3 écrans de cinéma, des groupes de haut-parleurs et ainsi de suite. Cette décision de l'Opéra de Cologne vexe profondément Zimmermann qui décide alors, selon ses propres termes, « d'entreprendre une grève afin de préserver la valeur de l'œuvre et la dignité de son créateur ${ }^{8}$.

Certains propos du compositeur permettent cependant d'envisager le pourquoi de cette pause forcée sous un autre angle. Dans une lettre au compositeur Markus Lehmann écrite à la fin de 1959, Zimmermann souligne, par exemple, que la composition de la deuxième scène de l'acte 2 constitue « un problème particulièrement épineux à résoudre $»^{9}$. La teneur du problème se situe dans la manière de réaliser - en musique - la représentation simultanée de trois actions séparées dans l'espace et le temps. L'examen des stratégies mises en œuvre à cette fin montre comment les choix musicaux effectués en 1959 ont pu à la fois entraîner la nécessité d'un recul et fournir les éléments de solution qui feront la spécificité conceptuelle et technique du pluralisme musical.

Fasciné depuis toujours par tout projet philosophique (saint Augustin, Bergson, Husserl, Heidegger), littéraire (Joyce, Pound) ou pictural (Klee, Ernst, Schwitters) qui interroge la "simultanéité du non-simultané », Zimmermann trouve dans la pièce Die Soldaten de Jakob Lenz un matériau qui lui semble prédestiné. De cette pièce, écrite en 1776 , il dira que « la conception

Komponist und sein Werk, 187-200.

7Lettre de Zimmermann à Michael Gielen, 10 février 1964, dans « Bernd Alois Zimmermann : correspondance à propos des Soldats », établie et traduite par Vincent Barras, Musica 88 : Die Soldaten / Bernd Alois Zimmermann, 134.

8Lettre de Zimmermann à Marion Rothärmel, 9 juin 1963, dans « Bernd Alois Zimmermann : correspondance à propos des Soldats », 134.

9 « Bei der 2. Szene galt es im übrigen besonders schwierige Probleme zu lösen. Aber die Szene haut hin - und wie ! »; lettre de Zimmermann à Markus Lehman, 28 décembre 1959, citée par Gruhn, "Zur Entstehungsgeschichte von Bernd Alois Zimmermanns Oper Die Soldaten », 12. 


\begin{tabular}{|c|c|c|c|c|}
\hline \multicolumn{2}{|c|}{ Sections (version finale) } & \multirow{2}{*}{$\begin{array}{l}\text { Désignations } \\
\text { (version finale) }\end{array}$} & \multirow[t]{2}{*}{ Plan de 1958} & \multirow{2}{*}{$\begin{array}{c}\text { Date de composition }{ }^{\mathrm{t}} \\
\text { Novembre } 1962\end{array}$} \\
\hline Preludio & $*$ & & & \\
\hline \multirow[t]{7}{*}{ Acte 1} & Introduzione* & & & $<20$ mai $1963^{x}$ \\
\hline & Scène 1 & Strofe & Acte 1 , scène 1 & < Avril 1959 \\
\hline & Scène 2 & Ciacona I & Acte 1 , scène 2 & < Avril 1959 \\
\hline & Tratto I & & & $<21$ septembre 1963 \\
\hline & Scène $3^{*}$ & Ricercari I & Acte 1 , scène 3 & < Avril 1959 \\
\hline & Scène 4 & Toccata & Acte 1, scène 4 & < Avril 1959 \\
\hline & Scène $5^{*}$ & Notturno I & Acte 1 , scène 5 & $<$ Avril 1959 \\
\hline \multirow[t]{4}{*}{ Acte 2} & Introduzione & & & $<13$ novembre 1963 \\
\hline & Scène 1 & Toccata I & Acte 2 , scène 1 & $<$ Novembre 1959 \\
\hline & Intermezzo* & & & Avril 1963 \\
\hline & Scène 2* & $\begin{array}{l}\text { Capriccio, } \\
\text { Corale, } \\
\text { Ciacona II }\end{array}$ & Acte 2, scène 2 & < Janvier 1960 \\
\hline \multirow[t]{7}{*}{ Acte 3} & Preludio & & & $<25$ novembre 1963 \\
\hline & Scène 1 & Rondino & Acte 2 , scène 3 & < Octobre 1959 \\
\hline & Scène 2 & Rappresentazione & Acte 2 , scène 4 & $<$ Octobre 1959 \\
\hline & Scène 3 & Ricercari II & Acte 2 , scène 5 & <Octobre 1959 \\
\hline & Romanza & & & Juin 1964 \\
\hline & Scène 4 & Notturno II & Acte 2 , scène 6 & Juin 1964 \\
\hline & Scène 5 & Tropi & Acte 2 , scène $\tau$ & Juin 1964 \\
\hline \multirow[t]{6}{*}{ Acte 4} & & & $\begin{array}{l}\text { Acte } 3 \text { (une seule } \\
\text { scène) }\end{array}$ & \\
\hline & Preludio & & & Novembre 1964 \\
\hline & Scène 1 & Toccata. III & & Novembre 1964 \\
\hline & Tratto II & & & Novembre 1964 \\
\hline & Scène 2 & Ciacona III & & Novembre 1964 \\
\hline & Scène 3 & Nottumo III & & $\begin{array}{l}\text { Novembre } 1964 \text { (montage des } \\
\text { bandes termine au début de 1965) }\end{array}$ \\
\hline
\end{tabular}

'La version finale a été créé le 15 février 1965. Les sections constituant la Vokalsinfonie (créée le 20 mai 1963) sont identifiees par un astérisque.

bLes sections écrites lors de la seconde phase compositionnelle de même que leurs dates de composition apparaissent en italique.

'Le symbole * $<$ * signifie que la section a été composée avant la date indiquée. 
dramaturgique [...], détachée de la règle [aristotélicienne] des trois unités, vient mêler plusieurs actions dans une sorte d'anticipation de la Danse des heures de la simultanéité de Joyce ${ }^{10}$. » Zimmermann se réfère bien sûr ici au célèbre Ulysse de James Joyce ${ }^{11}$. La présence de scènes simultanées dans les Soldats, dont l'acte 2 fournit - dans la pièce de Lenz comme dans l'opéra - le premier exemple, découle en grande partie de la mise en œuvre de procédés littéraires. Zimmermann doit cependant leur trouver un équivalent à la fois conceptuel et musical.

La métaphore de la "sphéricité du temps " (Kugelgestalt der Zeit), que Zimmermann déduit de ses lectures philosophiques et qu'il répète inlassablement à partir de 1960, fournira l'équivalent conceptuel. Cette métaphore vise à traduire une conception du temps comme perpétuelle simultanéité du passé, du présent et du futur et signifie que les directions du temps - du passé vers le futur et du futur vers le passé - sont interchangeables. La célèbre formule des Confessions de saint Augustin - véritable leitmotiv dans les écrits zimmermanniens - fournit une des sources de cette conception :

[...] Il y a trois temps, le présent du passé, le présent du présent, le présent du futur. Il y a en effet dans l'âme, d'une certaine façon, ces trois modes de temps, et je ne les vois nulle part ailleurs. Le récit du passé, c'est la mémoire, le présent du présent, c'est la vision, le présent du futur, c'est l'attente ${ }^{12}$.

Pour Zimmermann, les Soldats ne raconte pas une histoire dans un temps chronologique, mais rendent compte

d'une situation universelle dont l'origine se trouve dans un futur qui menace le passé. Le futur c'est l'anéantissement vers lequel nous tendons tous perpétuellement et irrévocablement. Le passé c'est le jeu combiné de l'appartenance à une classe sociale et des circonstances de vie auxquelles l'existence humaine est universellement soumise, un jeu où l'être est toujours perdant ${ }^{13}$.

La deuxième scène de l'acte 2 (intitulée Capriccio, Corale e Ciacona II) est cruciale dans l'opéra puisqu'elle dévoile les principaux agents dramaturgiques qui perdront les protagonistes. Cette scène concrétise également - et avant la lettre - le concept de "sphéricité du temps ». Comme le montre le tableau 2, trois actions coïncident ici, marquées $\mathrm{A}, \mathrm{B}$ et $\mathrm{C}$ dans le schéma.

Les deux premières scènes sont déjà dans un rapport de simultanéité dans la pièce de Lenz (Lenz, acte 2 , scène 3$)$. Il s'y passe ceci ${ }^{14}$ :

10Zimmermann, «Les Soldats [non daté] », traduit par C. Gaspar et C. Fernandez, Contrechamps 5 (1985): 41.

$11 \mathrm{~J}$ 'ai abordé la question de la réception de James Joyce par Zimmermann et ses contemporains dans «Le modèle joycien et la pensée musicale des années cinquante et soixante ", dans Bericht über den internationalen Kongreß der Gesellschaft für Musikforschung : Musik als Text. Freiburg 27.9.1.10.1993 (à paraître).

12Extrait du Livre XI des Confessions de saint Augustin, cité et traduit par Paul Ricœur, Temps et récit (Paris : Éditions du Seuil, 1983), 32.

13 Voir Zimmermann, «Les Soldats [non daté] », 42.

14Les résumés de l'intrigue sont adaptés du « Synopsis » rédigé par Maren Bode (traduction de Jacques Fournier) pour le livret accompagnant l'enregistrement Teldec 9031-72775-2 du Stuttgarter Staatstheater sous la direction de Bernhard Kontarsky, réalisé en 1991. 
Introduction

(mes. 1-13)

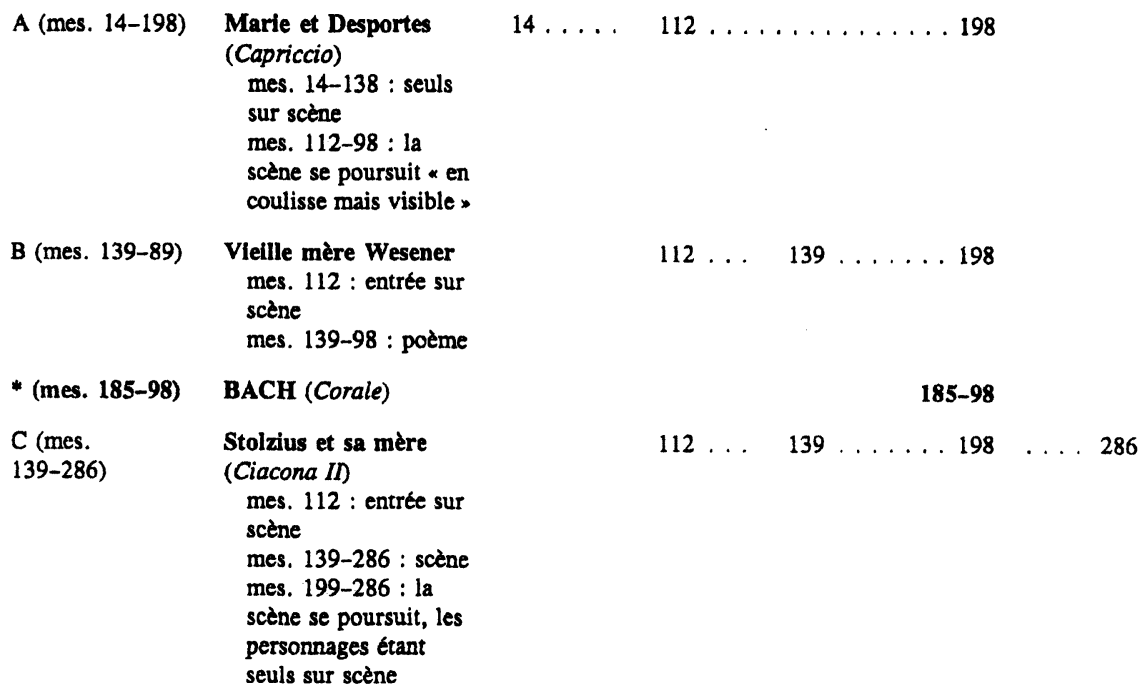

Tableau 2: Plan de l'acte 2, scène 2 de Die Soldaten

alors que Marie, sur le point d'écrire une lettre de rupture à son fiancé Stolzius, est séduite par Desportes (gentilhomme du Hainaut français et servant dans l'armée), sa grand mère (la vieille mère Wesener), récitant un poème prophétique (« La rose du Hainaut »), prévoit que les rires de Marie se changeront bientôt en larmes.

Zimmermann superpose ces deux actions à une troisième (Lenz, acte 3 , scène 2) :

Stolzius, relisant une lettre de Marie dans laquelle leur amour n'est pas encore mis en péril, la défend contre les accusations de sa mère qui la qualifie de « putain à soldats » et promet de se venger de Desportes.

Dans la réalisation musicale de cette simultanéité scénique, trois facteurs principaux montrent que les mécanismes qui feront la spécificité du pluralisme s'inscrivent directement dans la suite des stratégies développées ici :

(1) la conception de la scène comme construction polyphonique ; Zimmermann décrit en effet la scène comme un « triple contrepoint ${ }^{15}$.

(2) l'établissement d'une strate unificatrice citante (le $10^{\mathrm{e}}$ choral de la Passion selon saint Matthieu de Bach) servant de fondement dans un complexe impli-

15 «In der 2.Szene des gleichen Aktes der Oper werden drei Szenen (bei Lenz sind schon 2 dieser Szenen simultan miteinander verknüpft) gleichsam im dreifachen Kontrapunkt komponiert [...]»; Zimmermann, «Lenz und neue Aspekte der Oper, » blätter + bilder 9 (1960) :39-44 ; «Lenz, nouvelles perspectives pour l'opéra », traduit par Carlo Rossi, Contrechamps 5 (1985) : 39. 
quant trois éléments; dans le schéma comme dans la partition, cette strate est marquée Corale.

(3) le plan global de la scène faisant se juxtaposer ces trois éléments au centre d'une structure tripartite.

Le premier facteur anticipe la nature de toute l'entreprise pluraliste et procède d'une aspiration inlassable à garantir l'ordonnance du chaos. Pour Zimmermann, l'essence même de la règle contrapuntique généralisée (où styles, périodes historiques et médias deviennent des paramètres) fournit, avec la série, un principe d'ordre à la multiplicité. Pour justifier la mise en œuvre de ce principe, Zimmermann, comme plusieurs de ses contemporains, emploiera des modes de constructions polyphoniques éprouvés tels le cantus firmus ou le canon de proportions. Chez lui, l'usage de fragments liés historiquement à l'élaboration de ce type de structure - comme un choral ou une mélodie grégorienne -, viendra resserrer ce lien avec les techniques du passé.

L'histoire compositionnelle zimmermannienne révèle que le compositeur récupérera d'abord la technique du cantus firmus ${ }^{16}$. Dans la scène qui nous occupe, la citation du $10^{\mathrm{e}}$ choral de la Passion selon saint Matthieu ( "Ich bins, ich sollte büßen $»)^{17}$ sert de cantus firmus. La technique du cantus firmus deviendra un mode privilégié de constitution des passages citants dans des œuvres dénuées de toute prétention programmatique avouée ; on pense au rôle du Veni creator spiritus dans le concerto pour deux pianos et orchestre intitulé Dialoge $(1960,1965)$. Ici, ce n'est pourtant encore que la dramaturgie et la mise en scène qui exigent la présence d'une strate unificatrice.

Dans un article intitulé «Voix décalées », Markus Spies explique comment " l'absence d'une lettre provoque la redéfinition du poème prophétique de la vieille mère Wesener en rumeur ${ }^{18}$. " On se rappellera ici que Marie n'a pas écrit sa lettre de rupture à Stolzius. Le désespoir de Stolzius ne peut donc s'expliquer que par le fait qu'il est exposé, comme les autres, au bruit qui court selon lequel Marie est devenue une « putain à soldats ». Pour Spies,

au pont dramaturgique poème-rumeur-auditeur [...] correspond une catégorie musicale spécifique dont l'évocation nous rappelle que nous devons nousmêmes être des auditeurs : nous ne pouvons faire autrement que d'extraire de la trame des voix le choral qui y est tissé. Stolzius est forcé d'entendre, comme nous sommes forcés d'entendre ${ }^{19}$.

Cette interprétation de l'importance dramaturgique de la citation du choral (elle s' « impose » à l'auditeur) renforce le caractère citationnel et référentiel

16 On consultera à ce sujet le chapitre 4 ( «La citation génératrice de procédés compositionnels ») de ma thèse «La citation dans la pensée créatrice de Bernd Alois Zimmermann », 141-49.

17 Il s'agit ici de la première "citation de répertoire " des Soldats. Par « citation de répertoire " j'entends un fragment emprunté à la musique dite " sérieuse », par opposition aux emprunts d'ordre stylistique que constituent les épisodes de jazz dans la scène 1 de l'acte 2 ("Danse de l'Andalouse ») et les scènes 1 et 3 de l'acte 4.

18 Markus Spies, « Voix décalées, » traduit par Ole Hansen-Love, Musica 88 : Die Soldaten / Bernd Alois Zimmermann, 109-11.

19 Ibid., 97. 
du poème de la vielle mère Wesener (« La rose du Hainaut ») qui, il est important de le rappeler, est déjà présent dans le texte de Lenz ${ }^{20}$. Pour Spies, " le travail de l'écriture [au sens dramaturgique] est restitué sous forme de citation ". D'un point de vue strictement musical, l'insertion du choral s'est avérée tout aussi efficace. L'analyse du passage montre en effet que c'est le choral comme strate unificatrice qui autorise la juxtaposition des trois plans musicaux en présence. La mise en musique définit une structure tripartite où, comme le montre le tableau 2, seule la section centrale fait se juxtaposer les trois actions ; une superposition enveloppée par la résonance du choral de Bach que Zimmermann cite au complet ${ }^{21}$. Le « triple contrepoint » dont parle Zimmermann n'est donc que momentané.

Dramaturgiquement, c'est la vieille mère Wesener qui, dès son entrée sur la scène (mes. 112), détient le véritable rôle d'agent de la simultanéité. Le mode d'insertion du choral - qui maximise les facteurs symbiotiques - vient confirmer ce rôle dans le musical. Les exemples 1 et 2 confirment d'abord l'appartenance de la mélodie du choral et de la mélodie de la vieille mère à une même strate temporelle caractérisée, si on la compare aux métriques et aux rythmes sérialisés des sections $A$ et $C$, par le cours uniforme et plus lent du temps. L'exemple 1 reproduit la mise en musique du premier vers chanté par la vieille mère (« Kindlein mein, O Kindlein mein », mes. 139-40). En utilisant le second hexacorde de la forme inversée et transposée de la série principale de la scène (série $7: 1-2$ ), ce vers anticipe incontestablement la tonalité du choral de Bach, la bémol majeur. L'exemple 2 montre la structure rythmique analogue (voire l'identité dans le cas des premières mesures) des deux mélodies.

Quant à la configuration même du passage citant (dont l'exemple 3 reproduit la partition, mes. 185-92), elle découle pour ainsi dire de la structure du choral cité aux vents et aux cordes. On note, entre autres, que chacune des phrases du choral correspond à une phrase des protagonistes et est articulée, comme elles, par une pause du discours. La coïncidence fréquente entre les hauteurs des parties vocales et les hauteurs du choral, favorisée par une segmentation hexacordale de la série mettant en évidence des hauteurs clés (voir tout particulièrement la partie vocale de la vieille mère, mes. 187-90), vient corroborer le rôle unificateur de la citation et le haut degré d'intégration souhaité ici.

Cette volonté d'intégration renvoie aux techniques plus anciennes d'insertion d'une citation unique, tissée au discours ambiant ou encore à la source même de ce discours. Cependant, le rôle de strate temporelle et la position de la citation au centre d'une structure tripartite symétrique (A : dialogue, Marie et Wesener / B : monologue de la vieille mère Wesener / C : dialogue, Stolzius et sa mère) anticipent la technique d'insertion des passages à citations désormais multiples dans des œuvres comme Dialoge $(1960,1965)$ et Photoptosis (1968) et dans l'Intermezzo de l'acte 2 des Soldats composé en 1963. La

20Mes tentatives de trouver l'origine (littéraire et musicale) de ce poème dans un chant populaire sont demeurées infructueuses.

21 Dans les sections de l'opéra et les œuvres composées après ce passage, Zimmermann ne citera plus d'extrait complet : il s'agira toujours de fragment au sens littéral du terme. 


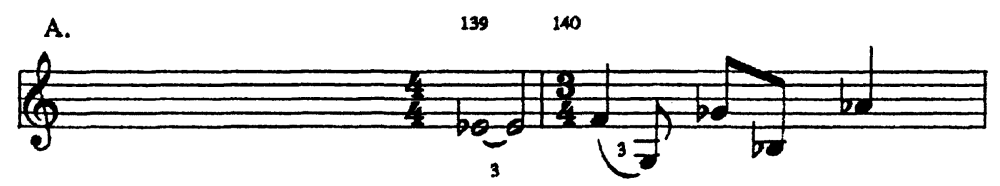

B.

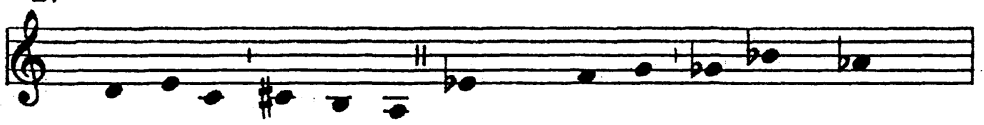

Exemple 1: Bernd Alois Zimmermann, Die Soldaten. a) acte 2, scène 2, mes. 139-40 (vers initial du poème de la vieille mère Wesener, « La rose du Hainaut »); b) série 7 des Soldats : 1-2.

a.

153

154

155

156

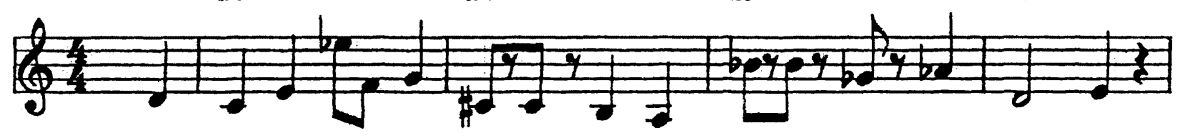

b.

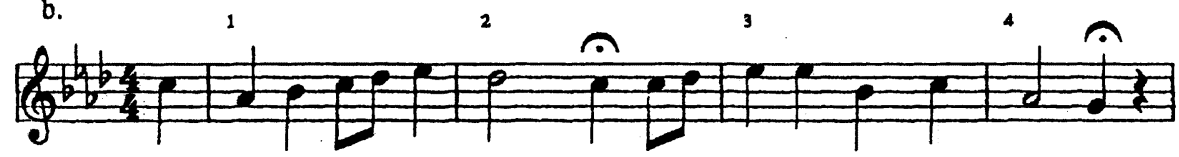

Exemple 2: Bernd Alois Zimmermann, Die Soldaten, acte 2, scène 2. a) auatre premières mesures de la mélodie de la vieille mère Wesener (mes. 153-56), correspondant à la première strophe de son poème « La rose du Hainaut »; $b$ ) quatre premières mesures du choral « Ich bin's ich sollte büßen » (cité aux mes. 185-89).

citation est donc ici sur le point de changer de statut. Le caractère transitoire des stratégies observées dans cette scène autorise donc, à mon sens, l'établissement de ce passage comme prototype de la technique zimmermannienne du pluralisme musical.

\section{«Intermezzo »}

La préparation d'un concert où seront présentés, le 20 mai 1963, des extraits des Soldats donne le coup d'envoi à la seconde phase compositionnelle de l'opéra. Cette seconde phase, incontestablement pluraliste, constitue l'aboutissement des expériences effectuées dans les œuvres composées pendant l'interruption comme Dialoge (terminé en septembre 1960), Présence (terminé en mars 1961) et Antiphonen (terminé en janvier 1962). Les deux autres passages citants de l'opéra que je propose d'examiner brièvement ici ont été composés pour cette occasion : le « Preludio » (en novembre 1962) et l' «Intermezzo » de l'acte 


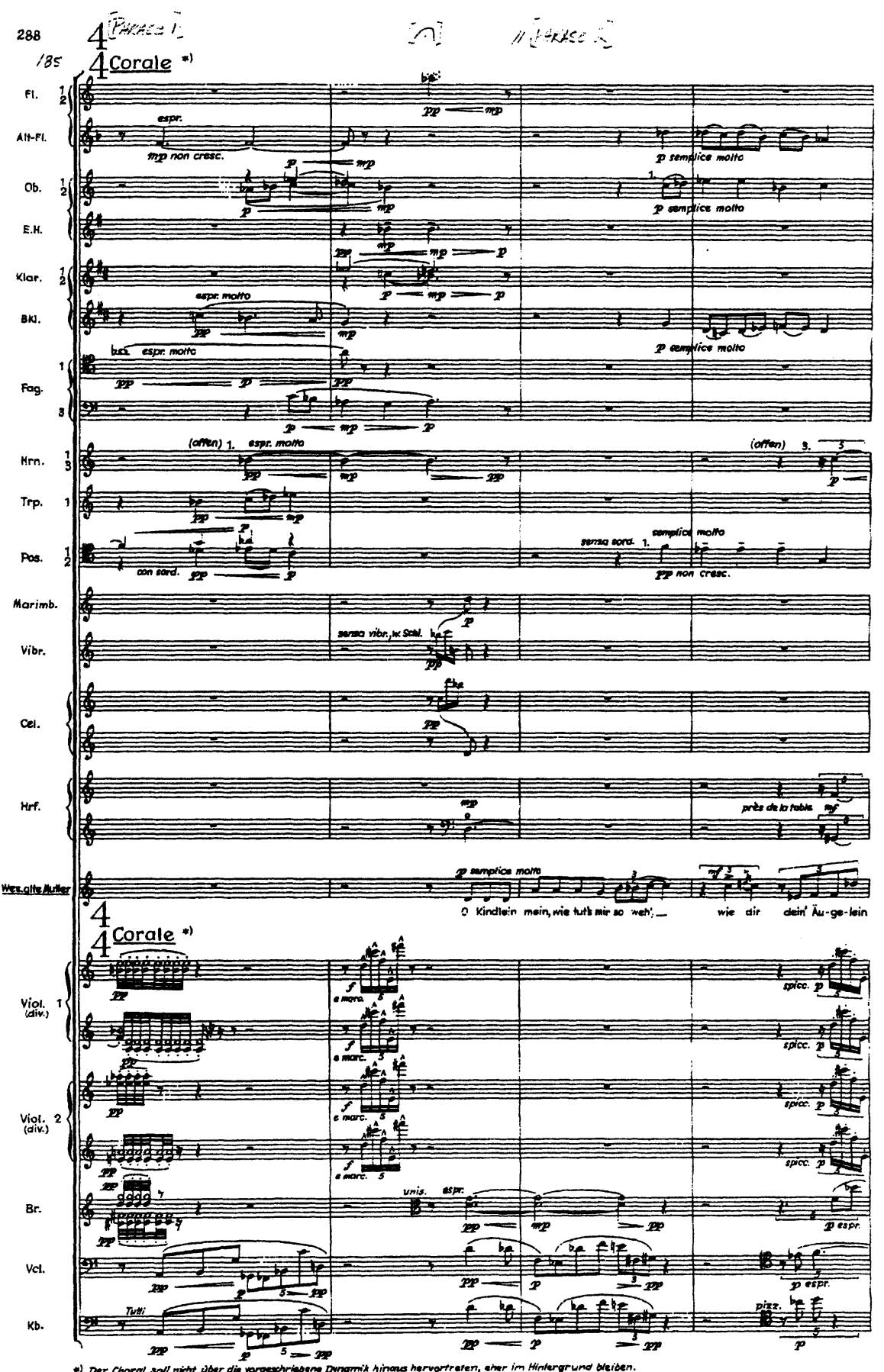

Exemple 3: Bernd Alois Zimmermann, Die Soldaten, acte 2, scène 2, mes. 185-92. (1) B. Schott's Söhne, Mayence, 1975. Reproduit avec l'autorisation de la European American Music Distributors Corporation. 


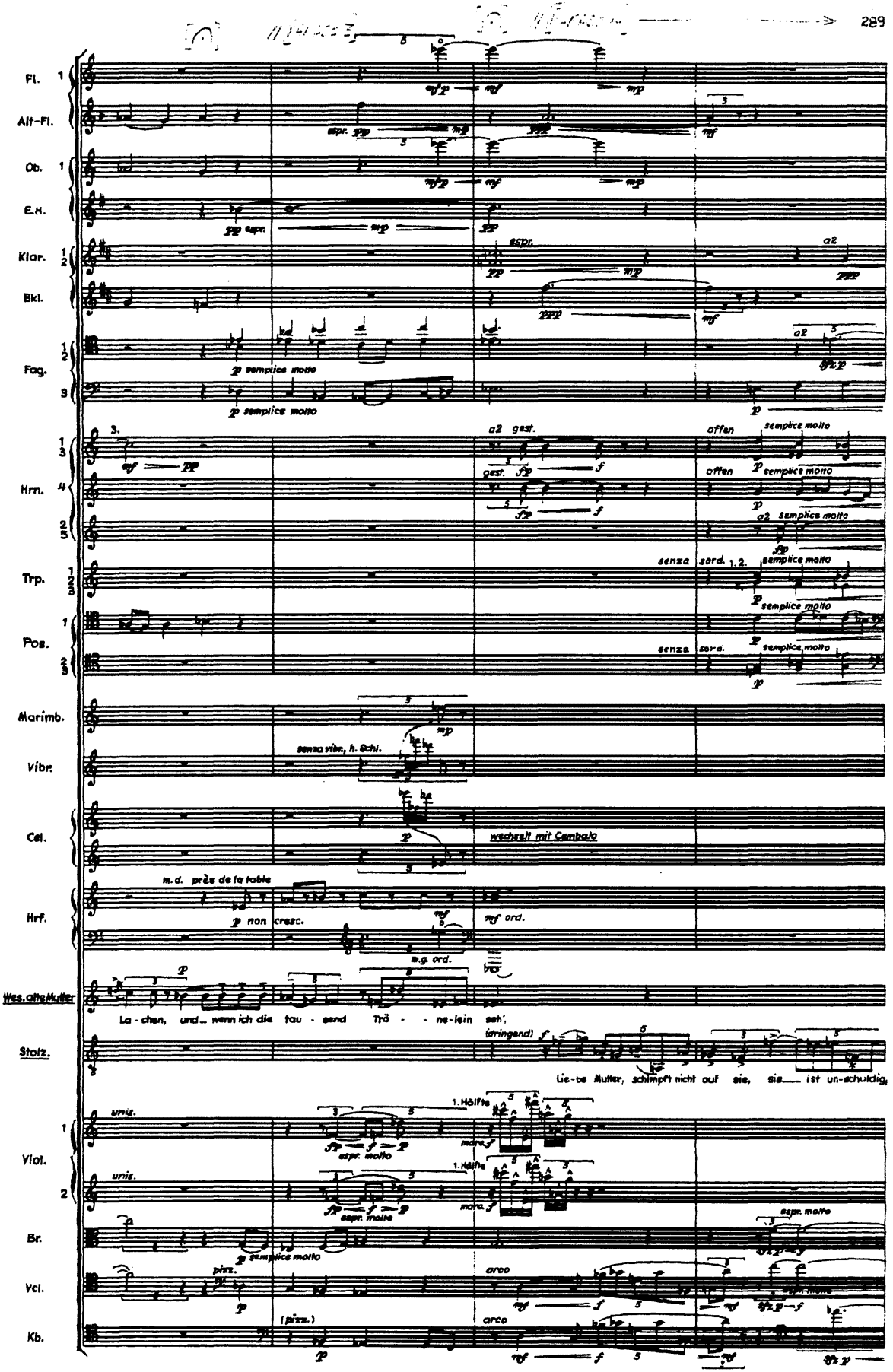

Exemple 3: (suite) 
2 (en avril 1963) ${ }^{22}$. Mais voyons d'abord comment Zimmermann définit les rouages techniques de la composition pluraliste et quel rôle il attribue désormais à la citation. L'extrait d'un texte publié en 1968 résume le projet :

Cette théorie [la sphéricité du temps] implique, du point de vue de la stricte technique de composition, le choix pour une œuvre ou pour un ensemble d'œuvres, d'un complexe contraignant de hauteurs (généralement une série de douze sons utilisant tous les intervalles) qui engendre une structure proportionnelle constituée de diverses couches de temps. Celles-ci correspondent strictement, dans leur durée effective, au complexe de sonorités choisi ce qui n'empêche pas l'insertion spontanée de d'autres musiques, présentes ou futures, des citations ou de simples collages ${ }^{23}$.

C'est la multiplicité temporelle qu'autorisent la conception sphérique du temps et le principe de stratification fondateur de l'œuvre qui permet l'insertion de fragments ; une multiplicité qui demeure cependant soumise à l'ordre dicté par une série. Dans tous les passages citants répertoriés à partir de la troisième période créatrice (pluraliste), les fragments cités seront insérés suivant le code rigoureux qui détermine l'ensemble des relations qui s'opèrent dans l'œuvre. Chaque fragment cité constituera une strate temporelle possédant son tempo et sa durée propres, établissant de ce fait un rapport précis avec une autre strate, et ce selon la série préétablie 24 . La facture de l' «Intermezzo » de l'acte 2 des Soldats montre de quoi il s'agit.

Bien entendu, la contiguiité de l' «Intermezzo » et de la deuxième scène du deuxième acte favorise la création de liens symboliques et structurels entre les deux sections. Ainsi, la structure de l' « Intermezzo » est tripartite et sa section centrale citante met trois éléments en présence. Mais la fusion des éléments ne constitue plus le but premier. Les citations sont maintenant collées les unes aux autres et seul le rapport de proportion métrique et temporel (durée des fragments), déduit des proportions intervalliques qui caractérise la série fondatrice, garantit la cohérence du discours.

22Lors de ce concert du 20 mai 1963, Zimmermann réunira sous le titre Die Soldaten : Vokalsinfonie, les scènes 3 et 5 de l'acte 1, la scène 2 de l'acte 2 (trois scènes déjà composées), le « Preludio ", l'« Introduzione » de l'acte 1 et l' « Intermezzo » de l'acte 2 (trois sections composées pour l'occasion). Voir le Tableau 1.

23 « Das bedeutet, rein kompositionstechnisch gesehen, daß aus einer für ein ganzes Werk oder für eine ganze Werkgruppe verbindlichen Tonhöhenkonstellation (meistens einer Allintervallreihe) ein Proportionsgefüge von verschiedenen Zeitschichten abgeleitet wird, die auf der einen Seite in ihrer effektiven Zeitdauer auf das Strengste mit der erwähnten Tonhöhenkonstellation verbunden sind, auf der anderen Seite aber durch die Möglichkteit spontaner Einbeziehung von vergangener oder zukünftiger Musik, von Zitaten oder Zitatcollagen [...]. Zimmermann, « Vom Handwerk des Komponisten [1968] », dans Intervall und Zeit : Bernd Alois Zimmermann, Aufsätze und Schriften zum Werk (Mayence : Schott, 1974), 35 ; « Du métier de compositeur », traduit par C. Caspar et C. Fernandez, Contrechamps 5 (1985) : 58 [traduction revue].

24La série des rapports de proportions intervalliques générés par la série de hauteurs choisie sera transformée en une série de proportions temporelles exprimée sous forme de tempos. Le rapport 4:5 caractérisant la tierce majeure ascendante, par exemple, est équivalent au rapport noire $=90$ : noire $=$ 114. Ces mécanismes que Zimmermann décrit, entre autres, dans son article "Intervall und Zeit " (publié pour la première fois dans la Frankfurter Allgemeine Zeitung en mai 1957) sont très proches de ceux qu'expose Stockhausen dans son célèbre « wie die Zeit vergeht... » paru la même année (Die Reihe, $n^{\circ} 3$ [1957]). 
La première section de l' "Intermezzo » (section A) installe un rapport de proportion métrique qu'actualisent deux strates temporelles non-citantes dans un rapport s'approchant de 6:5 (c'est-à-dire une tierce mineure) : 4/8, croche $=$ env. 90 (cuivres, Bühnenmusik, percussion et orgue) $/ 4 / 8$, croche $=$ env. 107 (xylophone, vibraphone, marimba). La section centrale (section B) se divise en deux parties (l'exemple 4 reproduit les quatre premières mesures de la première partie).

La première partie de cette section centrale fait entendre trois strates dont deux sont citantes (mes. 21-33) :

(1) les trombones, le tuba et les cloches transposent des extraits du choral «Wenn ich einmal soll scheiden » de la Passion selon saint Matthieu de Bach dans le registre grave et en augmentation rythmique. Il s'agit ici de la cinquième et dernière apparition du choral principal dans la Passion selon saint Matthieu.

(2) le Dies ira est démultiplié (canon) à l'orgue en une polyphonie panisorythmique à trois voix : la voix supérieure et la voix inférieure font entendre les trois versets de la séquence, alors que la voix médiane présente les versets 1 et 2 . Chacune des voix possède sa talea propre et dans toutes les voix les strophes sont doublées comme le veut la pratique d'exécution médiévale de la séquence. Il s'agit ici de la réinsertion du canon qui clôt le " Preludio ", composé presqu'en même temps (bien que les talea en aient été modifiées) et dont il sera question plus loin.

(3) le xylophone, le vibraphone et le marimba exécutent une figure en septolets, issue de la série principale de la deuxième scène du premier acte (« Ciacona I » : série 2 des Soldats, P-2). Tout au long de l'opéra cette série sera associée au personnage de Stolzius.

Dans la seconde partie de la section centrale de l' «Intermezzo » (dont le début est reproduit à l'exemple 5), trois strates sont de nouveau en présence, alors que le choral de Bach de la première partie se poursuit jusqu'à la mes. 39 :

(1) aux trompettes : deux extraits du choral pour orgue « Komm Gott Schöpfer, heiliger Geist », dont la mélodie d'origine est celle de l'hymne Veni creator spiritus $^{25}$.

(2) Bühnenmusik : rythme de marche.

(3) au glockenspiel, au vibraphone et aux cloches : sorte de trio engendré par la série 11 des Soldats (tricordes chromatiques) rappelant une des deux strates de la première section de l' «Intermezzo ».

Dans le premier collage (première partie de la section), la nature rythmique de chaque strate établit une hiérarchie où le choral de Bach agit comme strate fondatrice sur laquelle prolifèrent le Dies irae (en valeurs courtes et se démultipliant lui-même) et la figure en septolets (toujours un peu plus élaborée). La

25On notera ici que le Veni creator spiritus constitue le cantus firmus des sections citantes de Dialoge et de Photoptosis. 


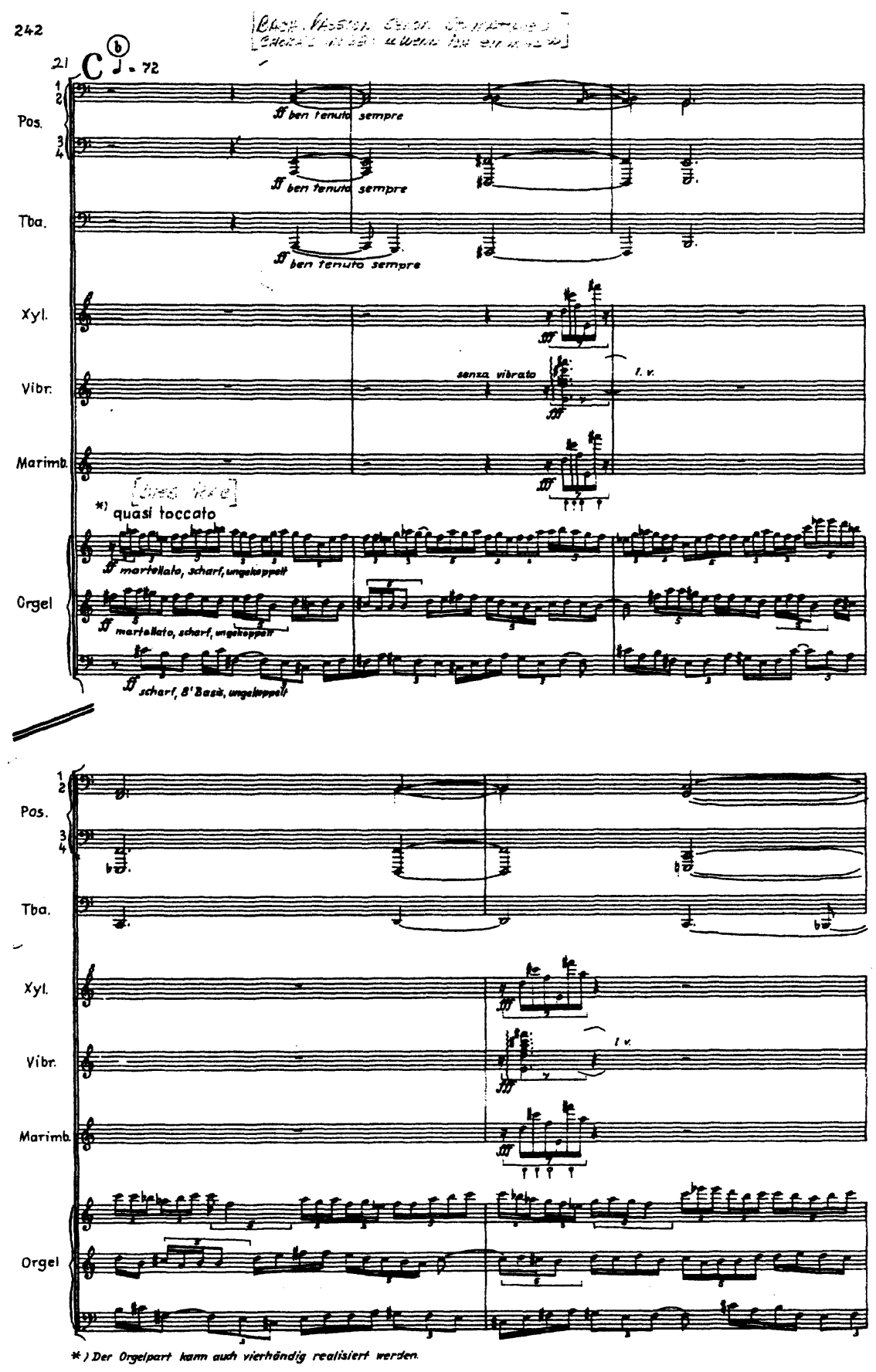

Exemple 4: Bernd Alois Zimmermann, Die Soldaten, acte 2, «Intermezzo », mes. 21-25. () B. Schott's Söhne, Mayence, 1975. Reproduit avec l'autorisation de la European American Music Distributors Corporation. 


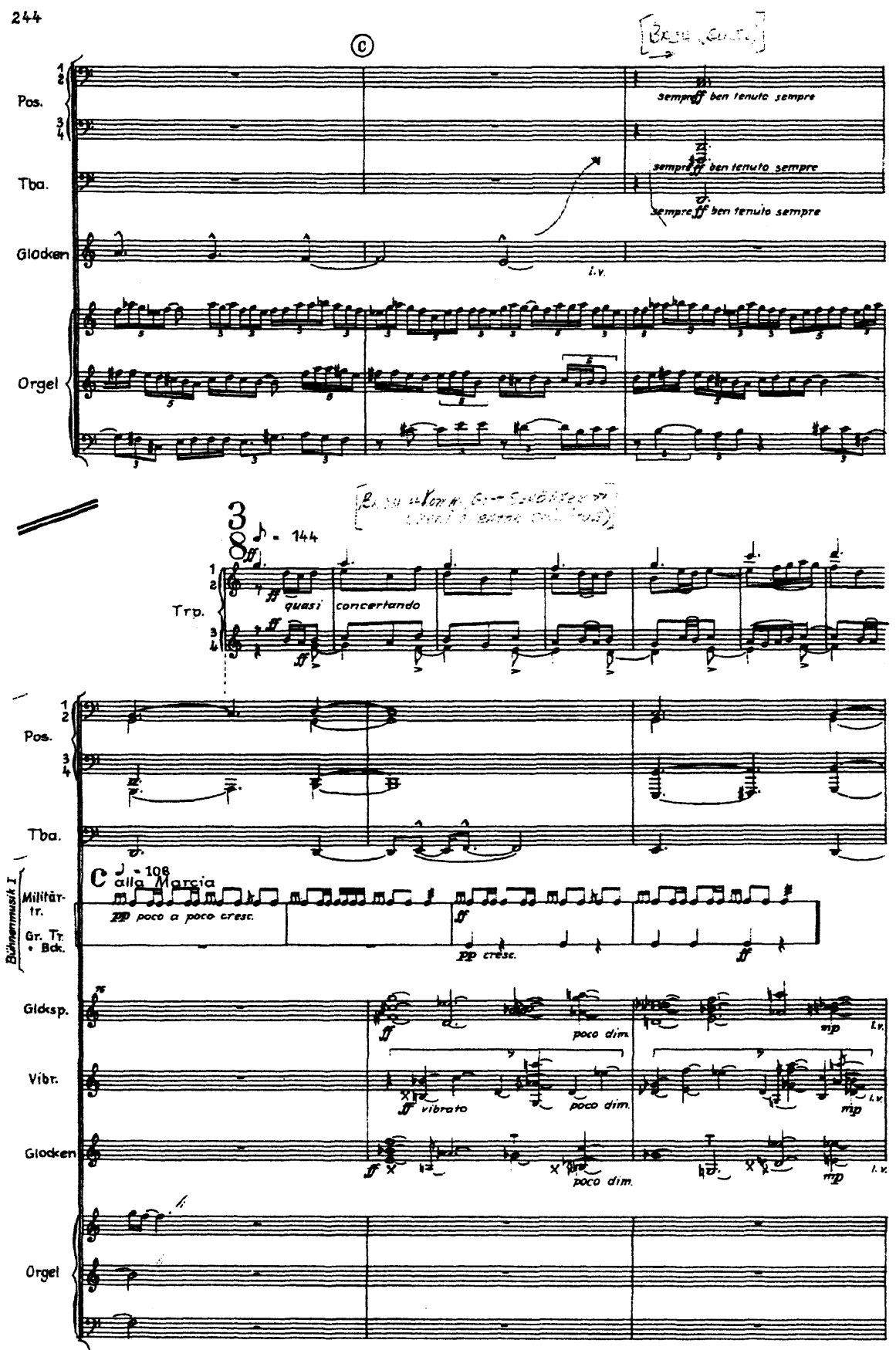

Exemple 5: Bernd Alois Zimmermann, Die Soldaten, acte 2, «Intermezzo », mes. 30-35. (C) B. Schott's Söhne, Mayence, 1975. Reproduit avec l'autorisation de la European American Music Distributors Corporation. 
prolongation du choral de Bach dans le second collage (seconde partie) semble confirmer ce rôle.

On observe donc ici, de nouveau, une stratification par trois et citante au centre de la structure, préparant, dans l'opéra, à celle qui sera donnée à entendre dans la scène qui suit (acte 2 , scène 2 ) et dont il a été question plus haut. Cette stratégie constitue un des deux principaux modes d'insertion des citations observés chez Zimmermann à partir de la période pluraliste ${ }^{26}$. Dans cet «Intermezzo ", la démultiplication du Dies irae en canon isorythmique à trois voix intensifie la symbolique du chiffre trois dans toute l'œuvre de Zimmermann mais surtout, pour notre propos, renvoie au second mode d'insertion des fragments cités : le canon de proportion.

\section{«Preludio »}

À partir de la troisième période créatrice les canons abondent dans les partitions de Zimmermann et constituent un mode privilégié de construction des microstructures. Comme mode de constitution de passage à citation unique et monodique, ces textures canoniques, dont la proximité avec les micropolyphonies de Ligeti ${ }^{27}$ ne peut nous échapper, se chargent de sens. Comme dans le cas de la dilatation temporelle d'un cantus firmus, la démultiplication et le maniement rythmique complexe - impliquant la différenciation par superposition de proportions temporelles - d'une seule ligne mélodique en un nombre élevé de voix $(24,36,48)$ brouille la notion du temps chez l'auditeur, transgresse la perception de l'objet original ${ }^{28}$ et permet la représentation simultanée d'une multitude d'expériences temporelles, voire de la multiplicité tout court. La mise en scène par démultiplication du Dies ira dans la section conclusive du

26Dans Photoptosis [Irruption de lumière], par exemple, l'insertion du collage de citations - Veni creator spiritus (comme cantus firmus), Beethoven/Scriabine, Wagner/Zimmermann, Bach/Tchaikovsky) - vient imposer une forme extérieure tripartite au processus formel. La mise en présence de deux strates principales dans les première et troisième sections et l'organisation de la section médiane citante (présence d'une strate unificatrice / hiérarchisation des strates / établissement de rapports proportionnels spécifiques entre les segments empruntés, stratification privilégiant la présence simultanée de trois éléments comparés au rapport par deux des sections encadrant la section médiane) montre l'importance des stratégies développées dans la scène 2 de l'acte 2 et dans l' «Intermezzo » du même acte des Soldats.

27On notera, au passage, la similitude des intentions de Ligeti et de Zimmermann dans l'exploitation des techniques canoniques. Pour Ligeti, le canon à l'unisson permet "l'unité de la succession et de la simultanéité, de l'horizontal et du vertical » et " offre la possibilité de composer une toile de fils mélodiques selon des règles de constructions assez bien définies. "Voir Pierre Michel, György Ligeti, compositeur aujourd'hui (Paris : Minerve, 1985), 151-52.

28Pierre Boulez décrit ainsi cette notion fondamentale : « L'identité, l'identification plutôt, de l'objet musical au travers des multiples modifications qu'on est amené à lui faire subir, n'a guère été mise en doute : tous les compositeurs se sont servis abondamment, dans les contextes les plus traditionnels, des techniques de l'augmentation et de la diminution littérale, du renversement, du rétrograde, des déplacements harmoniques, que sais-je encore ! L'arsenal est vaste, il remonte aux origines mêmes de notre polyphonie. Et cependant cette polyphonie elle-même n'a-t-elle pas pour origine une transgression de la perception? Le cantus firmus allongeant à l'extrême dans le temps une mélodie destinée à être " comprise" - aux deux sens de ce terme - dans les limites les plus restreintes, à une vitesse “normale " n'a-t-il pas déjà rendu méconnaissable l'objet initial ? [...] On sent chez l'" inventeur" le désir et le besoin de se référer à un objet initial ; son travail, son plaisir, voire sa malice consisteront bien souvent à égarer ou à aider l'auditeur, quant à la connaissance ou la reconnaissance de cet objet initial à travers les transformations qu'il lui impose, aux dérivées qu'il en tire. » Pierre Boulez, Jalons (pour une décennie) (Paris : Christian Bourgois, 1989), 301-2. 
«Preludio » des Soldats poursuit ce but (l'exemple 6 reproduit les quatre premières mesures de la section finale).

La densité maximum maintenue presque continuellement permet d'envisager l'ensemble du «Preludio » comme l'analyse composée d'un immense cluster, mettant d'ores et déjà les protagonistes en face de la catastrophe qui les menace. Le ré scandé inexorablement constitue un des seuls points de repère dans cette mer sonore privée de toute possibilité de résolution/réconciliation. La démultiplication du Dies irae dans la dernière section du "Preludio » confirme cette impossibilitée ${ }^{2}$. Malgré la présence d'un matériau unique, sa dispersion en 36 voix différenciées rythmiquement brouille toutes les pistes, même celle qui faisait du ré une anticipation du mode original (dorien) du Dies irae : le canon n'est pas à l'unisson mais couvre trois fois le total chromatique ${ }^{30}$. Débarrassé de sa fonction introductive traditionnelle (mise en place d'une atmosphère, énoncé-catalogue), le "Preludio » constitue d'emblée un point fort de l'opéra. Pour emprunter à Markus Spies,

avant le commencement du drame, le prélude désigne la dispersion du sens comme la catastrophe vers laquelle il court [...] l'œuvre ne fait pas de retour à un sens qui pourrait être affirmé, qui serait fixe. Et c'est la figure de la différence n'autorisant plus aucun rapprochement, qui devient de manière en quelques sorte corpusculaire le personnage dramatique principal des scènes ${ }^{31}$.

La tension entre la dispersion du sens (représentée soit par le canon de proportion, soit par la mise en présence momentanée de plusieurs citations renvoyant inévitablement hors du texte) et la volonté utopique de représenter un monde fini (par la « sphéricité du temps » et son équivalent, la série contenant tous les intervalles) constitue le fondement et le paradoxe de l'entreprise pluraliste de Zimmermann. Le caractère prétendu universel des fragments choisis (des chorals de Bach ou encore de la séquence de la messe des morts), est venu rendre ce paradoxe plus sensible. En reconnaissant la citation, l'auditeur est plus susceptible de percevoir la simultanéité des temps différents ${ }^{32}$.

${ }^{29} \mathrm{La}$ stratégie observée ici est très proche de celle que Zimmermann met en œuvre à la fin du Requiem für einen jungen Dichter (« Dona nobis pacem »), terminé quatre ans après l'achèvement des Soldats, où un chant très connu des mouvements ouvriers allemands (Brüder, zur Sonne, zur Freiheit) est également démultiplié en canon de proportion.

30Dans l'organisation globale de cette section, on discerne quatre strates principales clairement identifiées par l'instrumentation. La première (14 premiers violons, 12 seconds violons, 10 altos) fait entendre un canon à 36 voix, la deuxième un canon à trois voix à l'orgue (débutant réellement à la mes. 135), alors que la troisième est constituée de clusters se désintégrant peu à peu aux contrebasses et la quatrième, de ponctuations aux gong et à la caisse roulante. La première strophe du Dies irce fournit le matériau mélodique du canon aux cordes (mes. 126-50). Le canon à l'orgue (mes. 135-45) est un canon proportionnel à trois voix et fait entendre le Dies irce dans trois transpositions différentes : $f a, s i$ et $f a$. Le canon se distingue de celui entendu aux cordes en ceci que sa voix supérieure fait entendre les trois strophes de la séquence et non pas seulement la première.

31 Spies, «Voix décalées ", 105.

32Carl Dahlhaus a rappelé, en ce sens, comment dans le canon de proportion du second Agnus de la messe L'homme armé de Josquin des Prés - véritable passage fétiche auquel Zimmermann fait souvent allusion dans ses écrits -, " on perçoit les différentes couches de temps précisément par le fait que la même substance mélodique est à la base de toutes les voix ". Voir Carl Dahlhaus, "Sphéricité du temps : à propos de la musique de Bernd Alois Zimmermann ", traduit par Vincent Barras, Contrechamps 5 (1985) : 88. 
10

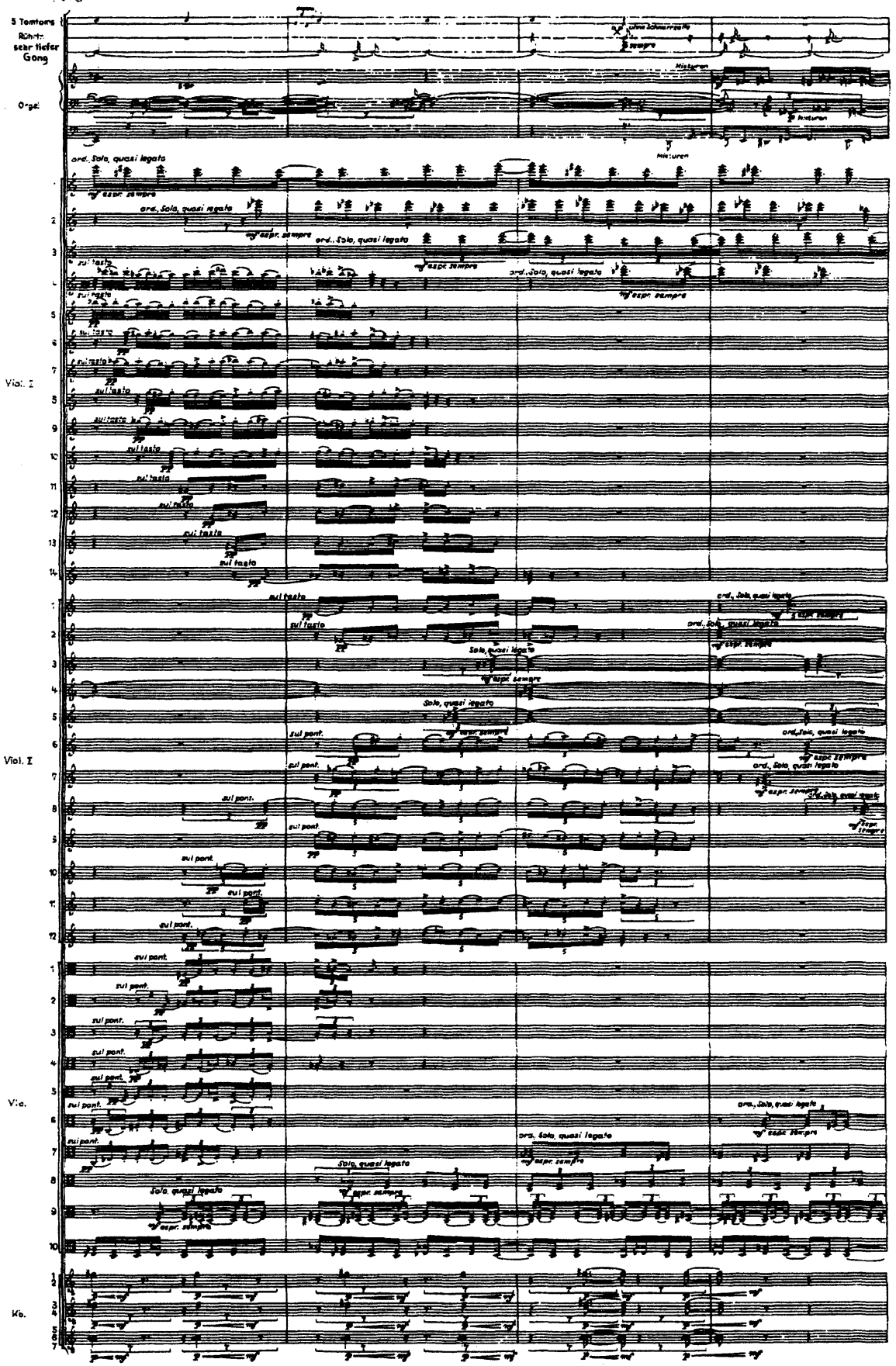

Exemple 6: Bernd Alois Zimmermann, Die Soldaten, « Preludio », mes. 126-129. (C) B. Schott's Söhne, Mayence, 1975. Reproduit avec l'autorisation de la European American Music Distributors Corporation. 
L'ouverture du discours à la charge émotive de la citation (la citation en référant l'auditeur à un champ acoustique différent et en faisant appel à sa mémoire surprend toujours) est venue, quant à elle, confirmer le rôle stratégique des passages citants dans la logique de la forme globale, soit dans son centre, soit comme aboutissement d'un processus.

Les stratégies compositionnelles que la présence de citation a engendrées ont bientôt conquis tous les paliers de composition (citants ou non) et ont contribué à mettre en péril le pouvoir longtemps irréfutable de la linéarité et de la finalité temporelle dans l'œuvre d'art. Chez Zimmermann, pour qui « c'est dans la victoire remportée sur le temps que se fonde le bonheur du compositeur $»^{33}$, la citation s'est avérée une arme efficace.

\section{Résumé}

L'auteure examine trois extraits de l'opéra Die Soldaten (1958-60, 1962-64) de Bernd Alois Zimmermann et montre comment la citation est venue fournir des solutions aux problèmes compositionnels rencontrés la dans conception d'une œuvre dramaturgique à ce moment crucial dans l'évolution vers la formulation du pluralisme musical, technique compositionnelle définie par le compositeur vers 1960 et visant à traduire une conception sphérique du temps. Ces trois passages sont la deuxième scène du deuxième acte (janvier 1960), où l'insertion d'un choral de Bach est venue résoudre le problème de la représentation simultanée de trois scènes, «Intermezzo » du même acte (avril 1963), privilégiant également un choral de Bach comme fondement du complexe (à la manière d'un cantus firmus) et enfin, le « Preludio » (novembre 1962), où la démultiplication du Dies ira en canon proportionnel révèle à la fois la fascination de Zimmermann pour les techniques polyphoniques franco-flamandes et l'importance structurelle qu'accorde le compositeur aux passages citants, ici non plus au centre d'une structure tripartite comme dans les deux cas précédents, mais comme aboutissement du processus dramaturgique.

33 «In der Überwindung der Zeit liege für ihn das Glück des Komponierens. » Propos recueillis en 1967 par Ursula Stürzbecher, « Bernd Alois Zimmermann », Werkstattgespräche mit Komponisten (Cologne : Hans Gerig, 1971), 154. 